\title{
Hierarchically acting sterile neutrinos
}

\author{
Chian-Shu Chen ${ }^{1, \mathrm{a}}$, Ryo Takahashi ${ }^{2, \mathrm{~b}}$ \\ ${ }^{1}$ Physics Division, National Center for Theoretical Sciences, Hsinchu, 300 Taiwan \\ ${ }^{2}$ National Tsing Hua University, Hsinchu, 300 Taiwan
}

Received: 26 April 2012 / Revised: 28 June 2012 / Published online: 25 July 2012

(C) The Author(s) 2012. This article is published with open access at Springerlink.com

\begin{abstract}
We propose that a hierarchical spectrum of sterile neutrinos $\left(\mathrm{eV}, \mathrm{keV}, 10^{13-15} \mathrm{GeV}\right)$ is considered as the explanation for MiniBooNE and LSND oscillation anomalies, dark matter, and baryon asymmetry of the universe (BAU), respectively. The scenario can also realize the smallness of active neutrino masses by the seesaw mechanism.
\end{abstract}

\section{Introduction}

The compelling evidence from solar, atmospheric, reactor, and accelerator neutrino experiments has established the phenomenon of neutrino oscillations. The standard description is that the experimental data can be nicely explained by the mixings between the flavor and mass eigenstates of the three neutrinos in Standard Model (SM), the so-called "active" neutrinos. The unitary mixing matrix is parameterized in terms of three rotation angles $\left(\theta_{12}, \theta_{23}, \theta_{13}\right)$ and one Dirac $C P$ violating phase $\delta_{C P}$. The probabilities of flavor oscillations are governed by the $\theta_{i j}$ and two masssquared differences $\Delta m_{12}^{2} \simeq 7.59 \times 10^{-5} \mathrm{eV}^{2}$ and $\left|\Delta m_{31}^{2}\right| \simeq$ $2.45 \times 10^{-3} \mathrm{eV}^{2}$ [1], where $\Delta m_{23}^{2}>0$ or $\Delta m_{23}^{2}<0$ refers to normal or inverted mass hierarchy spectrum, respectively. One of the most famous approaches to generate the active neutrino masses is the so-called "Type-I seesaw mechanism" [2-7], in which one adds $N$ right-handed neutrinos $N_{R_{i}}(i=1-N)$ to the $\mathrm{SM}$ and the active neutrino masses can be obtained by block diagonalizing the mass matrix of left- and right-handed neutrinos,

$m_{v_{\alpha \beta}}=-\sum_{i=1}^{N} \frac{M_{D_{\alpha i}} M_{D_{i \beta}}^{T}}{M_{R_{i}}}$.

Here $\alpha, \beta=e, \mu, \tau$ represent the flavor indices of the SM fermions, $M_{D}$ is the Dirac mass matrix formed through the

\footnotetext{
a e-mail: chianshu@phys.sinica.edu.tw

be-mail: ryo.takahasi88@gmail.com
}

Yukawa interactions between left- and right-handed neutrinos, and $M_{R_{i}}$ are the Majorana masses of right-handed neutrinos. Since the right-handed neutrinos are completely neutral under the SM gauge symmetries, the Majorana mass $M_{R}$ is a gauge invariant quantity and $N_{R_{i}}$ are often termed "sterile" neutrinos. At least two sterile neutrinos are needed to accommodate the two mass splits observed experimentally. The mass scales of $M_{D_{\alpha i}}$ and $M_{R_{i}}$ are free parameters and cannot be fixed by oscillation experiments alone.

There are, however, results from the LSND [8] and the MiniBooNE [9] which cannot be accommodated in three active neutrinos description and may need to introduce one or more sterile neutrinos at the $\mathrm{eV}$ scale to fit the data (e.g. see $[10,11])$. Moreover, possibility of the presence of light sterile neutrinos have been discussed in cosmology (e.g. see $[12,13])$.

Meanwhile, there is an increase in the amount and precision of cosmological data indicating that about $80 \%$ of the matter content in the universe is non-baryonic dark matter (DM). The study of nature of DM is one of the main topics in cosmology, astrophysics, and particle physics. Cold Dark Matter (CDM) is widely studied because the WIMP (weakly interacting massive particle) may reveal its signal at LHC and is predicted in many popular models (supersymmetric models, etc.). However, it has been noticed that a sterile neutrino with mass at the $\mathrm{keV}$ scale and with small mixing to the active neutrinos can make up the DM in the form of Warm Dark Matter (WDM) [14, 15]. Additionally, it was pointed out that the $\mathrm{keV}$ sterile neutrino might play an important role in explaining the pulsar kicks [16].

Finally, the level of one out of ten billions excess in the amount of matter over antimatter is a long standing puzzle for high energy physicists. The observation hints that baryon number $(B)$ and/or lepton number $(L)$ are violated in certain physical processes. Grand unified theories (GUTs) naturally provide a framework for breaking $B$ and $L$, in which the fundamental fermions-quarks and leptons-are arranged in the same multiplets, and the out-of-equilibrium decays of 
heavy gauge bosons or colored Higgs bosons $H_{C}$ will generate the sufficient baryon asymmetry around the scale of grand unification [17-23]. It was then recognized that the Standard Model (SM) violates $B+L$ symmetry through the $S U(2)_{L}$ global anomaly [24, 25]. The process is not suppressed during the period $100 \mathrm{GeV} \lesssim T \lesssim 10^{12} \mathrm{GeV}$, and the solution is called "sphalerons" [26]. The sphaleron effect violates $B+L$ but conserves $B-L$, and therefore, it would erase any primordial $B+L$ asymmetry. We notice that any grand unification theories with higher symmetries respects $B-L$ symmetry. For example, $B-L$ is a global symmetry for $S U(5)$ GUTs and a local symmetry for $S O$ (10) GUTs, respectively. The GUT-baryogenesis, therefore, is not able to explain baryon asymmetry in our universe (BAU). To solve the problem one has to generate $B-L$ asymmetry by violating pure baryon number [27-30] or by violating pure lepton number [31] (leptogenesis, e.g.), and the sphaleron process will convert partially $B-L$ asymmetry into baryon asymmetry. We adopt the construction that one heavy sterile neutrino causes lepton asymmetry during the epoch the sphalerons are ineffective, and the late decay of colored Higgs will generate the observed BAU.

\section{Hierarchically acting sterile neutrinos}

We consider a scenario of three sterile neutrinos $N_{R_{i(i=1-3)}}$ with hierarchical mass spectrum $\left(M_{R_{1}} \sim \mathrm{eV}, M_{R_{2}} \sim \mathrm{keV}\right.$, $M_{R_{3}} \sim 10^{13-15} \mathrm{GeV}$ ), in which the lightest one $N_{R_{1}}$ may help to explain the neutrino oscillation anomalies, $\mathrm{keV}$-scale sterile neutrino $N_{R_{2}}$ is the candidate of dark matter, and the heaviest state $N_{R_{3}}$ would resurrect the GUT-baryogenesis. Three sterile neutrinos can be introduced to cancel the additional gauge anomaly for any theory beyond SM with extra gauge $U(1)_{B-L}$ symmetry. We show this hierarchical spectrum of sterile neutrinos simultaneously satisfy the observations, and how our scenario fits in the framework of GUT theories.

It has been proposed that models of SM with (three) additional sterile neutrinos are phenomenologically viable [32-35]. ${ }^{1}$ The so-called $v$ MSM ( $v$ Minimal Standard Model) [32, 33], in which a mass of a keV sterile neutrino is responsible for DM, and two heavier states with degenerate masses lain in the range $1 \sim 100 \mathrm{GeV}$ are required to be in thermal equilibrium around electroweak scale in order to generate BAU through the resonant neutrino oscillations. The split seesaw model with three sterile neutrinos living in the extra dimension (ED) is shown to be able to solve DM and BAU as well [34]. By utilizing an exponential factor in the size of ED one can split the Majorana masses of $N_{R_{i}}$ with relative mild parameters associated to their locations

\footnotetext{
${ }^{1}$ See also [36-48] for recent related discussions.
}

Table 1 The content of three sterile neutrinos models

\begin{tabular}{lllll}
\hline Models & $\mathrm{eV}$ & $\mathrm{keV}$ & $\mathrm{GeV}$ & $\gg \mathrm{EW}$ \\
\hline$\nu$ MSM & & $N_{R_{1}}$ & $N_{R_{2}}, N_{R_{3}}$ & \\
Split Seesaw & & $N_{R_{1}}$ & & $N_{R_{2}}, N_{R_{3}}$ \\
BRZ & $N_{R_{1}}, N_{R_{2}}$ & $N_{R_{3}}$ & & \\
HASN & $N_{R_{1}}$ & $N_{R_{2}}$ & & $N_{R_{3}}$ \\
\hline
\end{tabular}

in ED. Recently a flavor symmetry model [35] proposed by Barry, Rodejohann, and Zhang (BRZ), it consists of two $N_{R_{1,2}}$ masses at eV scale and one $N_{R_{3}}$ at $\mathrm{keV}$ scale. The two eV-scale sterile neutrinos are used to explain LSND and MiniBooNE anomalies while the $\mathrm{keV}$ sterile neutrino is the WDM particle. The scenarios are summarized in Table 1 . These setup can answer two of the three puzzles we mentioned above while our hierarchically acting sterile neutrinos (HASN) scenario would explain the three puzzles simultaneously. The splittings of the sterile neutrino masses can be achieved by implementing split seesaw mechanism [34] or Froggatt-Nielsen (FN) mechanism [49] to the model. ${ }^{2}$

The Lagrangian which is relevant to neutrino masses has the form

$$
\begin{aligned}
\mathcal{L}= & \mathcal{L}_{\mathrm{SM}}+i \overline{N_{R_{i}}} \not \partial N_{R_{i}}-y_{\alpha i} H^{\dagger} \bar{l}_{\alpha} N_{R_{i}}-\frac{M_{R_{i}}}{2} \bar{N}_{R_{i}}^{c} N_{R_{i}} \\
& + \text { h.c. }
\end{aligned}
$$

Here $\mathcal{L}_{\mathrm{SM}}$ is the SM Lagrangian, $l_{\alpha}$ are $S U(2)_{L}$ leptonic doublets with flavor index $\alpha, H$ is SM Higgs, $y_{\alpha i}$ are the Yukawa couplings, and $c$ is charged conjugation. The Majorana mass matrix of sterile neutrinos is chosen to be diagonal without loss of generality. The $6 \times 6$ neutrino mass matrix is given in the form

$\left(\begin{array}{cc}0 & M_{D} \\ M_{D}^{\dagger} & M_{R}\end{array}\right)$

in the basis $\left(v_{e}, v_{\mu}, v_{\tau}, N_{R_{1}}, N_{R_{2}}, N_{R_{3}}\right)$, and $M_{R}=$ $\operatorname{diag}\left(\mathcal{O}(\mathrm{eV}), \mathcal{O}(\mathrm{keV}), \mathcal{O}\left(10^{13-15}\right) \mathrm{GeV}\right)$. Here we give a brief comment on a realization of such a hierarchical mass spectrum of right-handed neutrinos. One of simple examples to realize it is to utilize the split seesaw mechanism, in which the spinor fields are introduced in a flat five dimensional (5D) spacetime whose compactification length of extra dimension is $\ell$ and all SM particle are assumed to live in a 4D-brane. After solving the 5D Dirac equation and identifying the zero-modes of the 5D spinors with the righthanded neutrinos, the effective (4D) right-handed Majorana

\footnotetext{
${ }^{2}$ Since the scales of the sterile neutrino masses are very different, it might not unreasonable to consider that they have a different origin. For example, if an electroweak singlet Higgs boson exists and has a mass and VEV of the order of the electroweak scale, then its VEV can give the Majorana mass to one of the sterile neutrinos [50, 51].
} 
masses are described by exponential functions as

$M_{R i}=\frac{2 \kappa_{i} m_{i} v_{\mathrm{B}-\mathrm{L}}}{M\left(e^{2 m_{i} \ell}-1\right)}$,

where $\kappa_{i}, m_{i}, v_{\mathrm{B}-\mathrm{L}}$, and $M$ are a coupling constant of order one, bulk masses for $5 \mathrm{D}$ spinors, $U(1)_{\mathrm{B}-\mathrm{L}}$ breaking scale, and 5D fundamental scale, respectively. In this mechanism, one can easily obtain a hierarchical righthanded neutrino mass spectrum such as $\left(M_{R_{1}}, M_{R_{2}}, M_{R_{3}}\right)=$ $\left(1 \mathrm{eV}, 1 \mathrm{keV}, 10^{13} \mathrm{GeV}\right)$ within a set of moderate parameters when one takes $\kappa_{i}=1, v_{\mathrm{B}-\mathrm{L}}=10^{15} \mathrm{GeV}$, and $\left(M \ell, m_{1} \ell, m_{2} \ell, m_{3} \ell\right)=(30,27.9,24.4,1.03)$ as reference values. The FN mechanism can also give a hierarchical mass spectrum with appropriate $U(1)_{\text {FN }}$ charges.

After electroweak symmetry breaking where Higgs develops its vacuum expectation value (VEV) $v=174 \mathrm{GeV}$, one gets Dirac neutrino mass terms. The left-handed neutrinos receive their Majorana masses through seesaw mechanism, we obtain

$m_{v_{3}} \sim\left\{\begin{array}{ll}m_{\mathrm{atm}} \simeq \frac{\left|y_{\alpha 3}^{*} y_{\beta 3}\right| v^{2}}{M_{R_{3}}} & \text { for } \mathrm{NH} \\ \epsilon \simeq \frac{\left|y_{\alpha 2}^{*} y_{\beta 2}\right| v^{2}}{M_{R_{2}}} & \text { for } \mathrm{IH}\end{array}\right.$,

$m_{\nu_{2}} \sim m_{\mathrm{sol}} \simeq \frac{\left|y_{\alpha 1}^{*} y_{\beta 1}\right| v^{2}}{M_{R_{1}}} \quad$ for both $\mathrm{NH}$ and $\mathrm{IH}$,

$m_{v_{1}} \sim \begin{cases}\epsilon \simeq \frac{\left|y_{\alpha 2}^{*} y_{\beta 2}\right| v^{2}}{M_{R_{2}}} & \text { for } \mathrm{NH} \\ m_{\mathrm{atm}} \simeq \frac{\left|y_{\alpha 3}^{*} y_{\beta 3}\right| v^{2}}{M_{R_{3}}} & \text { for } \mathrm{IH}\end{cases}$

at the leading order, where $\mathrm{NH}$ and IH mean the normal hierarchy and inverted hierarchy, respectively. The indices $\alpha$ and $\beta$ in $m_{\nu_{3}}$ for NH should correspond to only $\mu$ and $\tau$ in order to be consistent with the current data of neutrino oscillation experiments, that is, there are a maximal atmospheric, a large solar, and a small reactor mixing angles. By choosing a set of appropriate values of the Yukawa couplings, the experimentally observed mixing angles can be always fitted in our scenario. Figure 1 shows our numerical fits of mixing angles, which can reproduce experimentally observed values as

$$
\begin{aligned}
& 7.12 \times 10^{-5} \mathrm{eV} \leq \Delta m_{21}^{2} \leq 8.20 \times 10^{-5} \mathrm{eV}, \\
& 2.26 \times 10^{-3} \mathrm{eV} \leq \Delta m_{21}^{2} \leq 2.77 \times 10^{-3} \mathrm{eV} \\
& 0.27 \leq \sin ^{2} \theta_{12} \leq 0.37 \\
& 0.39 \leq \sin ^{2} \theta_{23} \leq 0.64 \\
& 0.016 \leq \sin ^{2} \theta_{13} \leq 0.037
\end{aligned}
$$

at $3 \sigma$ level [52]. In the numerical fits, typical values of $\left|y_{\alpha i}\right|$ and $M_{R_{i}}$ are taken as discussed in this paper. The IH and degenerate mass spectra of active neutrinos can be also realized in the same manner.
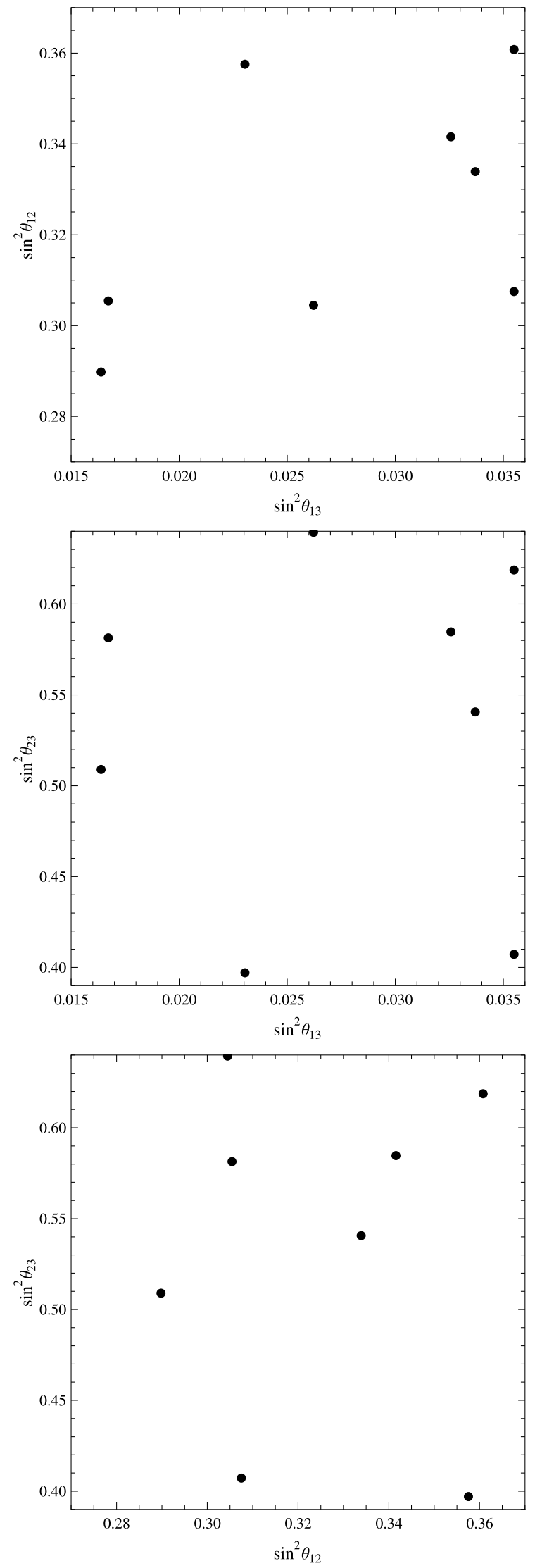

Fig. 1 Numerical fits of mixing angles in our scenario 
The dark matter candidate of the scenario is decaying DM. The keV sterile neutrino $N_{R_{2}}$ should live longer than the age of the universe and can be estimated as

$\tau_{N_{R_{2}}} \simeq 5 \times 10^{26}\left(\frac{M_{R_{2}}}{\mathrm{keV}}\right)^{-5}\left(\frac{10^{-8}}{\Theta^{2}}\right) \mathrm{s}$,

here $\Theta$ is the mixing between $\mathrm{keV}$ sterile neutrino and active neutrinos. We note that this mixing angle $\Theta$ is irrelevant to the explanations of LSND and MiniBooNE anomalies because only the lightest state of sterile neutrino $N_{R_{1}}$ plays a role for the explanations. We will discuss this point later. The generic way to produce DM is through the active-sterile neutrino oscillations [14], however, the abundance is constrained by the X-ray observations [53] (also see [54] and references therein), structure formation simulations [55], and the Lyman- $\alpha$ bounds [56, 57]. One way to relax the restrictions was proposed by Shi and Fuller [15] that an enhancement of the production of $\mathrm{keV}$ sterile neutrino can be realized via lepton-number-driven MSW (Mikheyev-Smirnov-Wolfenstein) effect. The other possibility is the $N_{R_{2}}$ pair production via $U(1)_{\mathrm{B}-\mathrm{L}}$ gauge boson exchange [34]. It has been shown that as long as the reheating temperature is about $10^{13} \mathrm{GeV}$ one can account for the relic abundance of DM. The corresponding Yukawa couplings of sterile neutrino DM for the required mass $\mathcal{O}(1) \mathrm{keV} \lesssim M_{R_{2}} \lesssim \mathcal{O}(10) \mathrm{keV}$ are typically restricted to $\mathcal{O}\left(10^{-15}\right) \lesssim\left|y_{\alpha 2}\right| \lesssim \mathcal{O}\left(10^{-13}\right)$ to satisfy astrophysical constraints (see e.g. [54] and references therein). This means that terms from the sterile neutrino DM through the seesaw mechanism does not contribute to the atmospheric and solar neutrino mass scales shown in (5)-(7). The Yukawa couplings for the first and third generations of right-handed neutrinos are approximated as $\left|y_{\alpha 3}^{*} y_{\beta 3}\right|^{1 / 2} \sim \mathcal{O}(0.1)$ and $\left|y_{\alpha 1}^{*} y_{\beta 1}\right|^{1 / 2} \sim \mathcal{O}\left(10^{-13}-10^{-12}\right)$ to satisfy the atmospheric and solar scales with $\left(M_{R_{1}}, M_{R_{3}}\right)=\left(1 \mathrm{eV}, 10^{13} \mathrm{GeV}\right)$, respectively. It is seen that the construction of active neutrino mass spectrum in HASN scenario is consistent with the constraints on $\mathrm{keV}$ sterile neutrino DM. It can be also found that the atmospheric scale can be derived from the ratio of the right-handed neutrino mass, $M_{R_{3}} \sim \mathcal{O}\left(10^{13}\right) \mathrm{GeV}$, and the corresponding Dirac masses, $\left|y_{\alpha 3}^{*} y_{\beta 3}\right|^{1 / 2} v \sim \mathcal{O}(10) \mathrm{GeV}$, when $N_{R_{3}}$ gets integrated out. While the solar scale comes from the seesaw relation between $M_{R_{1}} \sim \mathcal{O}(1) \mathrm{eV}$ and $\left|y_{\alpha 1}^{*} y_{\beta 1}\right|^{1 / 2} v \sim \mathcal{O}(0.1) \mathrm{eV}$. Finally, the ratio of mass scales between the second generation of sterile neutrino (DM), $M_{R_{2}} \sim \mathcal{O}(1) \mathrm{keV}$, and the corresponding Dirac masses, $\left|y_{\alpha 2}^{*} y_{\beta 2}\right|^{1 / 2} v \sim \mathcal{O}\left(10^{-3}-10^{-1}\right) \mathrm{eV}$, is too steep to contribute to the active neutrino mass (atmospheric and solar) scales. Therefore, the sterile neutrinos are hierarchically acting also for giving the active neutrino mass scales. The Yukawa structure realizing the scenario can be obtained in both split seesaw and FN mechanisms with appropriate model parameters.
Now we come to the phenomena of neutrino oscillation anomalies. The LSND $\bar{v}_{\mu} \rightarrow \bar{v}_{e}$ transitions anomaly reported a $3.8 \sigma$ excess of $\bar{v}_{e}$ candidate events, in which the neutrino fluxes were produced by dumping $800 \mathrm{MeV}$ protons into a "beam stop" which mostly generate $\pi^{+}$, and neutrinos (anti-neutrinos) are the decay products of pions. The probability that $v_{a}$ oscillates into $v_{b}$ is given by $P(a b)=\sin ^{2}(2 \theta) \sin ^{2}\left(1.27 \Delta m^{2} \frac{L}{E}\right)$, where $\theta$ is the mixing angle, $L$ is the neutrino travel distance in the unit of meter, and $E$ is the neutrino energy in MeV. The typical antineutrinos energies are a few $\mathrm{MeV}$ for reactor experiments, the excess is interpreted as the hints for $\bar{v}_{\mu} \rightarrow \bar{v}_{e}$ oscillation with $\Delta m^{2} \sim 1 \mathrm{eV}^{2}$. This indicates at least one sterile neutrino with mass at the $\mathrm{eV}$ scale. Then the MiniBooNE experiment set out to check the excess events in the $v_{\mu} \rightarrow v_{e}$ transitions and found the parameters were not compatible with LSND [58, 59]. However, more recently the MiniBooNE accumulated more anti-neutrino oscillation data and reported the excess electron anti-neutrino appearance is reconciled with LSND results [9]. To accommodate the neutrino and anti-neutrino data the additional CP violation has to be invoked. One simple way is to add two sterile neutrinos at $\mathrm{eV}$ scale (the so-called $(3+2)$ scheme) to neutrino sector, the $\mathrm{CP}$ violation at short-baselines would let to reconcile both LSND and MiniBooNE results [10, 11, 60-62]. Another way to explain the MiniBooNE results $[58,59]$ is a $(3+1)$ scheme together with nonstandard interactions (NSI) of neutrinos [10]. The new interactions may modify the charged and neutral currents, and provide the new sources of CP violation. They may affect the neutrino oscillations via the production, propagation, and the detection processes. The four-fermion operators can be expressed at low energies as

$$
\begin{aligned}
\mathcal{L}_{\mathrm{NSI}}= & 2 \sqrt{2} G_{F} \sum_{f} \epsilon_{\alpha \beta}^{f_{L, R}}\left(\bar{v}_{L \alpha} \gamma^{\mu} v_{L \beta}\right)\left(\bar{f}_{L, R} \gamma_{\mu} f_{L, R}\right) \\
& + \text { h.c., }
\end{aligned}
$$

where $G_{F}$ is Fermi constant, $f$ represents fermions (charged leptons and quarks), and $\alpha, \beta$ are flavor indices, and $L, R$ are chiralities. It has been shown that one can fit to global shortbaseline data for $\epsilon_{\alpha \beta} \sim \mathcal{O}\left(10^{-2}\right)$ [10]. For instance, the realization of CC-like interactions requires a charged particle inducing NSI. The value $\epsilon_{\alpha \beta} \sim \mathcal{O}\left(10^{-2}\right)$ expects the $\mathrm{TeV}$ scale mass of such particle but it is known that the NSI characterized by $\epsilon_{\alpha \beta} \sim \mathcal{O}\left(10^{-2}\right)$ can be hardly obtained from dimension- 6 operators without contradiction with bounds on charged lepton process [63,64]. Therefore, a $(3+1)$ with NSI scheme cannot be naturally embedded into our scenario. However, the latest update of analysis of shortbaseline neutrino oscillation data, which includes the recent update of MiniBooNE anti-neutrino data [65] and MINOS for $v_{\mu}$ disappearance into sterile neutrinos [66], in a $(3+1)$ without NSI scheme has pointed out that there are three regions within $1 \sigma$ at $\Delta m_{41}^{2} \simeq 1.6,1.2,0.91 \mathrm{eV}^{2}$ [67]. Note 
that $\Delta m_{41}^{2}$ almost corresponds to $M_{R_{1}}^{2}$ in our notation. The corresponding mixing angle among active neutrinos and the lightest sterile neutrino are of order $\mathcal{O}\left(10^{-2}-10^{-1}\right)$ to explain the anomalies. Those values can be realized by taking Yukawa coupling as $\left|y_{\alpha 1}\right| \sim \mathcal{O}\left(10^{-13}-10^{-12}\right)$, which are just consistent with the realization of active neutrino mass scales in our scenario as we mentioned above. Therefore, the $(3+1)$ without NSI scheme can be naturally embedded into our scenario. In this scheme, the second lightest sterile neutrino $N_{R_{2}}$, which can be a candidate for DM, and related mixing with active neutrinos does not affect on those discussions of anomalies because of the largeness of $M_{R_{2}}$ and smallness of mixing angles with active neutrinos.

It may be worth discussing the presence of such a light sterile neutrino in cosmology. One should note that such a light sterile neutrino is disfavored by considerations of hot dark matter (HDM), i.e., the sterile neutrino contributes to dark matter abundance too much [13]. But the bounds from the HDM on the sterile neutrino weaken if the cosmological framework of $\Lambda \mathrm{CDM}$ is modified. For instance, a dynamical dark energy model, which can generally lead to a equation of state parameter $\omega \neq-1$, with an additional relativistic degree of freedom can give a better fit than the one in the $\Lambda \mathrm{CDM}$ with three massless neutrino cosmological model [13]. For instance, a dark energy model as the mass varying neutrinos can accommodate the above ingredients (e.g. see [68-74]).

In the context of our consideration, we consider a GUTbaryogenesis via delayed decay of colored Higgs resurrected by Majorana interaction of heavy sterile neutrino [30]. The fundamental idea of this baryogenesis is as follows: In a GUT scale, the Yukawa interactions among quarks/leptons and colored Higgs are given by

$$
\begin{aligned}
\mathcal{L}_{Y}= & \psi(\mathbf{1 0})^{T} \lambda^{u(k)} \psi(\mathbf{1 0}) H_{C}^{(k)} \\
& +\psi(\mathbf{1 0})^{T} \lambda^{d(k)} \psi(\overline{\mathbf{5}}) \bar{H}_{C}^{(k)},
\end{aligned}
$$

for $k=1,2$ where we consider $S U(5)$ GUT for simplicity but it is straightforward to apply the scenario to other GUTs such as $S O(10)$ with or without supersymmetry. The baryon number can be generated by baryon number non-conserving decays,

$$
H_{c}^{(i)} \rightarrow l q, \quad H_{c}^{(i)} \rightarrow \bar{q} \bar{q},
$$

and their conjugates where $l$ stands for left-handed leptons $l_{L}$ and the right-handed charged lepton $e_{R}$, and $q$ denotes left-handed quarks $q_{L}$ and right-handed quarks $u_{R}$ and $d_{R}$. The baryon number can be produced by out-of-equilibrium decay of colored Higgs in the epoch of the Universe as $10^{12} \mathrm{GeV} \leq T \leq m_{H_{c}}$ where $T$ is the cosmic temperature and $m_{H_{c}}$ is a typical mass scale of colored Higgs particles. In this regime, inverse decays into the colored Higgs particles are blocked by the Boltzmann factor [19, 75, 76], and sphaleron transitions are ineffective. This is a scenario of GUT-baryogenesis via delayed decay of colored Higgs. There is, however, a crucial problem in this simple GUTbaryogenesis. It is that the process (16) and their conjugate conserve $B-L$. This means that all generated baryon number is erased by the sphaleron transitions at the temperature below $10^{12} \mathrm{GeV}$. However, if there is a lepton-numberviolating interaction at a temperature above $10^{12} \mathrm{GeV}$ and the interaction is in the thermal equilibrium, the interaction can resurrect this baryogenesis. Such interaction can be induced from the Majorana interaction of the sterile neutrino.

Once one adopts that a sterile neutrino $N_{R_{3}}$ is heavier than the colored Higgs bosons, and a lepton-numberviolating interaction $l \phi l \phi /\left(2 M_{R_{3}}\right)$, here $\phi$ is the Higgs doublet, all lepton asymmetry generated by colored Higgs decay is erased by the process $l+\phi \rightarrow \bar{l}+\phi^{\dagger}$ while the generated baryon asymmetry remains intact at the temperature above $10^{12} \mathrm{GeV}$. Therefore, $B-L \neq 0$ can be satisfied. When temperature drops below $10^{12} \mathrm{GeV}$ the sphaleron transitions become effective, as the results, the produced baryon asymmetry is partially converted into the lepton asymmetry but a residual baryon asymmetry remains, and thus the observed BAU can be generated. The resultant size of BAU is calculated as [21-23, 31, 77-80]

$Y_{\Delta B} \equiv \frac{n_{B}-n_{\bar{B}}}{s}=0.35 \cdot 0.5 \cdot 10^{-2} \cdot \frac{\epsilon_{B}}{1+(3 K)^{1.2}}$,

where

$\left.K \equiv \frac{1}{2} \frac{\Gamma}{H}\right|_{T=m_{H_{C}}} \simeq \frac{1.1 \times 10^{18} \mathrm{GeV}}{\lambda^{u 2} m_{H_{C}}}\left(\frac{1}{g_{*}}\right)^{1 / 2}$

is the washout factor and

$\epsilon_{B} \simeq \frac{\eta_{1}}{8 \pi} \cdot 10^{-2}[F(x)-F(1 / x)+G(x)-G(1 / x)]$

is the CP-asymmetry with $\Gamma, H, g_{*}, x$ are the decay rate, expansion rate, degrees of freedom $\left.g_{*}\right|_{T \simeq m_{H_{C}}} \simeq 53$, mass ratio $m_{H_{C}^{(2)}}^{2} / m_{H_{C}^{(1)}}^{2}$, respectively. The functions $F$ and $G$ are defined as $F(x) \simeq 1-x \ln \left(\frac{1+x}{x}\right)$ and $G(x)=\frac{1}{x-1}$. The factor 0.35 comes from the sphaleron process, and we take $\eta_{1}=\sin \left(\arg \left[\operatorname{tr}\left(\lambda^{d(1) \dagger} \lambda^{d(2)} \lambda^{u(1) \dagger} \lambda^{u(2)}\right)\right]\right)$ and $\eta_{1} \simeq \eta_{2}$. It is seen that we realize $Y_{\Delta B}=8.75 \times 10^{-11}$ when we set $\left(m_{H_{C}^{(1)}}, m_{H_{C}^{(2)}}\right)=\left(9 \times 10^{12}, 8 \times 10^{12}\right) \mathrm{GeV}$ and $\eta_{1} \simeq$ -0.444 . These values ${ }^{3}$ are consistent with this baryogenesis

\footnotetext{
${ }^{3}$ In a regime of such a relatively large value of washout factor, the decays of colored Higgs dominate over the pair annihilation processes such as $H_{c} \bar{H}_{c} \rightarrow q \bar{q}$ and $l \bar{l}$, etc. At higher temperature as $m_{H_{c}} \ll T$, the $2 \leftrightarrow 2$ scattering of the colored Higgs as $H_{c} \bar{H}_{c} \rightarrow q \bar{q}$ and $l \bar{l}$ etc. could also provide the colored Higgs production in addition to one from heavy gauge boson decay. The large value of washout factor also means those scatterings decoupled from equilibrium much earlier than the colored Higgs decay process.
} 
scenario and above discussion of the right-handed neutrinos mass spectrum, that is,

$10^{12} \mathrm{GeV} \leq m_{H_{C}^{(i)}}<M_{R_{3}} \lesssim 10^{15} \mathrm{GeV}$.

A heavier mass of the corresponding sterile neutrino as $10^{14-15} \mathrm{GeV}$ is also possible for this baryogenesis.

Finally we comment on a realization in $S O(10)$ case. In the $S O(10)$ case, the each generation of SM fermions and right-handed (sterile) neutrinos can be naturally included into a spinor 16-dimensional representation, $\psi(\mathbf{1 6}) .{ }^{4}$ The colored Higgs bosons, which play an important role in the above GUT-baryogenesis, is assigned into 10-plets in the $S O(10)$ case to give gauge invariant Yukawa interactions like in (15). We also need one $\mathbf{1 2 6}$ representation Higgs to obtain a Georgi-Jarlskog factor -3 , which give a realistic mass spectrum of charged leptons. There are some breaking chains of $S O(10)$ to the SM gauge group. For instance, if a 210-plet Higgs is introduced, the $S O(10)$ can be broken down to the Pati-Salam gauge group, $S U(4)_{C} \times S U(2)_{L} \times$ $S U(2)_{R}$.

\section{Summary and discussions}

The hierarchical spectrum of sterile neutrinos $(\mathrm{eV}, \mathrm{keV}$, $10^{13-15} \mathrm{GeV}$ ) is a simple and economical scenario, especially it can be embedded in many frameworks beyond SM. In light of the puzzles from neutrino oscillation anomalies, dark matter, and baryon asymmetry of the universe (BAU) we have shown that this scenario is phenomenologically viable. Regarding the active and sterile neutrinos oscillations, more precise data of MiniBooNE experiment and future data from reactors and the long baseline experiments might check the presence of the additional sterile neutrinos. For cosmological observation of $\mathrm{keV}$ sterile neutrino DM, X-ray observatory like the Chandra, which can search for an emission line from radiatively decaying DM, will be a strong tool for checking the $\mathrm{keV}$ sterile neutrino DM scenario. Finally, it is too difficult to test the baryogenesis in our scenario, and relate it to low energy phenomena.

Acknowledgement The research for this work has been supported in part by funds from the National Science Council of Taiwan under Grant Nos. NSC97-2112-M-006-004-MY3, NSC-98-2112-M-007-008-MY3 and the National Center for Theoretical Sciences and National Tsing Hua University.

\footnotetext{
${ }^{4}$ When one considers radiative corrections to the Majorana masses of right-handed neutrinos $\delta M_{R_{i}}$, there exist effects on the mass operator $\xi\left(H^{\dagger} H\right) \bar{N}_{R_{i}}^{c} N_{R_{i}}$ from the colored Higgs bosons $H_{c}$ at 1-loop level. Note that the colored Higgs bosons $H_{c}$ are propagating in the loop and a similar discussions have been given in [81]. In the case, the radiative correction to the Majorana mass are suppressed by heavy colored Higgs as $\delta M_{R_{i}}<\lambda m_{v}^{2} M_{R_{i}} / M_{H_{c}}^{2}$ where $\lambda$ is a 4-scalar coupling constant of order one and $m_{v}$ is a typical active neutrino mass scale.
}

Open Access This article is distributed under the terms of the Creative Commons Attribution License which permits any use, distribution, and reproduction in any medium, provided the original author(s) and the source are credited.

\section{References}

1. K. Nakamura et al. (Particle Data Group), J. Phys. G 37, 075021 (2010). And 2011 partial update for the 2012 edition

2. P. Minkowski, Phys. Lett. B 67, 421 (1977)

3. T. Yanagida, in Proceedings of the Workshop on Unified Theories and Baryon Number in the Universe, ed. by O. Sawada, A. Sugamoto (1979). KEK report 79-18

4. M. Gell-Mann, P. Ramond, R. Slansky, in Supergravity, ed. by P. van Nieuwenhuizen, D.Z. Freedman (North Holland, Amsterdam, 1979)

5. R.N. Mohapatra, G. Senjanovic, Phys. Rev. Lett. 44, 912 (1980)

6. J. Schechter, J.W.F. Valle, Phys. Rev. D 22, 2227 (1980)

7. J. Schechter, J.W.F. Valle, Phys. Rev. D 25, 774 (1982)

8. A. Aguilar et al. (LSND Collaboration), Phys. Rev. D 64, 112007 (2001)

9. A.A. Aguilar-Arevalo et al. (The MiniBooNE Collaboration), Phys. Rev. Lett. 105, 181801 (2010)

10. E. Akhmedov, T. Schwetz, J. High Energy Phys. 1010, 115 (2010)

11. J. Kopp, M. Maltoni, T. Schwetz, Phys. Rev. Lett. 107, 091801 (2011)

12. J. Hamann, S. Hannestad, G.G. Raffelt, I. Tamborra, Y.Y.Y. Wong, Phys. Rev. Lett. 105, 181301 (2010)

13. J. Hamann, S. Hannestad, G.G. Raffelt, Y.Y.Y. Wong, J. Cosmol. Astropart. Phys. 1109, 034 (2011)

14. S. Dodelson, L.M. Widrow, Phys. Rev. Lett. 72, 17 (1994)

15. X.-D. Shi, G.M. Fuller, Phys. Rev. Lett. 82, 2832 (1999)

16. A. Kusenko, G. Segre, Phys. Lett. B 396, 197 (1997)

17. M. Yoshimura, Phys. Rev. Lett. 41, 281 (1978). Erratum-ibid. D 42, 746 (1979)

18. S. Dimopoulos, L. Susskind, Phys. Lett. B 81, 416 (1979)

19. D. Toussaint, S.B. Treiman, F. Wilczek, A. Zee, Phys. Rev. D 19, 1036 (1979)

20. S. Weinberg, Phys. Rev. Lett. 43, 1566 (1979)

21. S.M. Barr, G. Segre, H.A. Weldon, Phys. Rev. D 20, 2494 (1979)

22. D.V. Nanopoulos, S. Weinberg, Phys. Rev. D 20, 2484 (1979)

23. A. Yildiz, P.H. Cox, Phys. Rev. D 21, 906 (1980)

24. G. 't Hooft, Phys. Rev. Lett. 37, 8 (1976)

25. G. 't Hooft, Phys. Rev. D 14, 3432 (1976). Erratum-ibid. 18, 2199 (1978)

26. V.A. Kuzmin, V.A. Rubakov, M.E. Shaposhnikov, Phys. Lett. B 155, $36(1985)$

27. J.M. Cline, S. Raby, Phys. Rev. D 43, 1781 (1991)

28. K.S. Babu, R.N. Mohapatra, S. Nasri, Phys. Rev. Lett. 97, 131301 (2006)

29. P.-H. Gu, U. Sarkar, Phys. Lett. B 705, 170 (2011)

30. M. Fukugita, T. Yanagida, Phys. Rev. Lett. 89, 131602 (2002)

31. M. Fukugita, T. Yanagida, Phys. Lett. B 174, 45 (1986)

32. T. Asaka, M. Shaposhnikov, Phys. Lett. B 620, 17 (2005)

33. T. Asaka, S. Blanchet, M. Shaposhnikov, Phys. Lett. B 631, 151 (2005)

34. A. Kusenko, F. Takahashi, T.T. Yanagida, Phys. Lett. B 693, 144 (2010)

35. J. Barry, W. Rodejohann, H. Zhang, arXiv:1110.6382 [hep-ph]

36. A. Boyarsky, A. Neronov, O. Ruchayskiy, M. Shaposhnikov, JETP Lett. 83, 133 (2006)

37. A. Boyarsky, J. Nevalainen, O. Ruchayskiy, Astron. Astrophys. 471, 51 (2007)

38. A. Boyarsky, D. Iakubovskyi, O. Ruchayskiy, V. Savchenko, Mon. Not. R. Astron. Soc. 87, 1361 (2008) 
39. A. Boyarsky, J. Lesgourgues, O. Ruchayskiy, M. Viel, Phys. Rev. Lett. 102, 201304 (2009)

40. H.J. de Vega, N.G. Sanchez, Mon. Not. R. Astron. Soc. Lett. 404, $885(2010)$

41. H.J. de Vega, N.G. Sanchez, Int. J. Mod. Phys. A 26, 1057 (2011)

42. H.J. de Vega, P. Salucci, N.G. Sanchez, arXiv:1004.1908 [astroph.CO]

43. I.M. Shoemaker, K. Petraki, A. Kusenko, J. High Energy Phys. 1009, 060 (2010)

44. D.K. Ghosh, G. Senjanovic, Y. Zhang, Phys. Lett. B 698, 420 (2011)

45. M. Lindner, A. Merle, V. Niro, J. Cosmol. Astropart. Phys. 1101, 034 (2011)

46. A. Merle, V. Niro, J. Cosmol. Astropart. Phys. 1107, 023 (2011)

47. A. Adulpravitchai, R. Takahashi, J. High Energy Phys. 1109, 127 (2011)

48. C.Q. Geng, R. Takahashi, arXiv:1201.1534 [hep-ph]

49. C.D. Froggatt, H.B. Nielsen, Nucl. Phys. B 147, 277 (1979)

50. A. Kusenko, Phys. Rev. Lett. 97, 241301 (2006)

51. K. Petraki, A. Kusenko, Phys. Rev. D 77, 065014 (2008)

52. D.V. Forero, M. Tortola, J.W.F. Valle, arXiv:1205.4018 [hep-ph]

53. K. Abazajian, G.M. Fuller, W.H. Tucker, Astrophys. J. 562, 593 (2001)

54. A. Boyarsky, O. Ruchayskiy, M. Shaposhnikov, Annu. Rev. Nucl. Part. Sci. 59, 191 (2009)

55. M.R. Lovell, V. Eke, C.S. Frenk, L. Gao, A. Jenkins, T. Theuns, J. Wang, A. Boyarsky et al., Mon. Not. R. Astron. Soc. 420, 2318 (2012)

56. S.H. Hansen, J. Lesgourgues, S. Pastor, J. Silk, Mon. Not. R. Astron. Soc. Lett. 333, 544 (2002)

57. M. Viel, J. Lesgourgues, M.G. Haehnelt, S. Matarrese, A. Riotto, Phys. Rev. D 71, 063534 (2005)

58. A.A. Aguilar-Arevalo et al. (The MiniBooNE Collaboration), Phys. Rev. Lett. 98, 231801 (2007)
59. A.A. Aguilar-Arevalo et al. (The MiniBooNE Collaboration), Phys. Rev. Lett. 102, 101802 (2009)

60. O.L.G. Peres, A.Y. Smirnov, Nucl. Phys. B 599, 3 (2001)

61. M. Sorel, J.M. Conrad, M. Shaevitz, Phys. Rev. D 70, 073004 (2004)

62. M. Maltoni, T. Schwetz, Phys. Rev. D 76, 093005 (2007)

63. S. Antusch, J.P. Baumann, E. Fernandez-Martinez, Nucl. Phys. B 810, 369 (2009)

64. M.B. Gavela, D. Hernandez, T. Ota, W. Winter, Phys. Rev. D 79, 013007 (2009)

65. E.D. Zimmerman (MiniBooNE Collaboration), arXiv:1111.1375 [hep-ex]

66. P. Adamson et al. (MINOS Collaboration), Phys. Rev. Lett. 107, 011802 (2011)

67. C. Giunti, M. Laveder, Phys. Rev. D 84, 093006 (2011)

68. R. Fardon, A.E. Nelson, N. Weiner, J. Cosmol. Astropart. Phys. 0410, 005 (2004)

69. R. Fardon, A.E. Nelson, N. Weiner, J. High Energy Phys. 0603, 042 (2006)

70. R. Takahashi, M. Tanimoto, Phys. Lett. B 633, 675 (2006)

71. R. Takahashi, M. Tanimoto, J. High Energy Phys. 0605, 021 (2006)

72. R. Takahashi, M. Tanimoto, Phys. Rev. D 74, 055002 (2006)

73. R. Takahashi, M. Tanimoto, Phys. Rev. D 77, 045015 (2008)

74. M. Honda, R. Takahashi, M. Tanimoto, J. High Energy Phys. 0601, 042 (2006)

75. S. Weinberg, Phys. Rev. Lett. 42, 850 (1979)

76. M. Yoshimura, Phys. Lett. B 88, 294 (1979)

77. J.N. Fry, K.A. Olive, M.S. Turner, Phys. Rev. Lett. 45, 2074 (1980)

78. J.N. Fry, K.A. Olive, M.S. Turner, Phys. Rev. D 22, 2977 (1980)

79. F.J. Botella, J. Roldan, Phys. Rev. D 44, 966 (1991)

80. J. Liu, G. Segre, Phys. Rev. D 48, 4609 (1993)

81. A. Aparici, A. Santamaria, J. Wudka, J. Phys. G 37, 075012 (2010) 